\title{
Nonlinear-Fuzzy Based Design Actuator Fault Diagnosis for the Satellite Attitude Control System
}

\author{
Alireza Mirzaee ${ }^{1}$ and Ahmad Foruzantabar ${ }^{2}$ \\ ${ }^{1}$ Highgradute in Control Engineering, \\ Electronic Department of Islamic Azad University-Dariun Branch, Shiraz-Iran \\ Mirzaee.edu@gmail.com \\ ${ }^{2}$ Phd Student in Control Engineering, \\ Electronic Department of Fars Science and Reseach Branch Islamic Azad University, \\ Marvdasht-Iran \\ A. foruzantabar@srbiau.ac.ir
}

\begin{abstract}
The objective of this paper is to develop a hybrid scheme (nonlinear observer and fuzzy decision making) for fault detection and isolation (FDI) in the reaction wheels of a satellite. Specifically, the goal is to decide whether a bus voltage fault, a current fault or a temperature fault has occurred in one of the three reaction wheels and further to localize which fault has occurred. In order to achieve these objectives, high fidelity model is used to exhibit the dynamics of the wheels on each of the three axes independently. First using the dynamic equations, nonlinear observer is designed, and then comparing estimated and actual states, residual signals are generated. These two input signals comprise the decision making unit. Design of the decision making unit using fuzzy reasoning is implemented. The effectiveness of this nonlinear-fuzzy based FDI scheme is investigated and a comparative study is conducted with the performance of a generalized observer-based scheme.
\end{abstract}

Keywords: Fuzzy decision making, qualitative reasoning, nonlinear observer, fault detection and isolation, fault diagnosis, reaction wheel, satellite, TakagiSugeno.

\section{Introduction}

Attitude control is an important basic function for most spacecrafts especially for satellites. It has been widely studied since late 1950s [1]. The attitude control subsystem stabilizes the spacecraft and orients it in the desired set point position in a short time despite the presence of external disturbance torques. The control torques could be formed from a combination of momentum wheels, reaction wheels, control moment gyros, thrusters or magnetic torquers. The main actuators for satellite attitude control systems are reaction wheels.

A high fidelity mathematical model of a reaction wheel [2] is discussed briefly in Section 2. Normally, there are three types of faults in a wheel that require special attention. The first is the bus voltage fault. The bus voltage should be sufficiently high to avoid elimination of the voltage headroom. Low bus voltage will result in reduced torque capacity and consequently cause serious instability of the satellite attitude. The same effect will appear when the motor current loss occurs in the wheel this leads to a 
loss of power and therefore the wheel cannot supply enough reaction torque to achieve a proper set point change of the attitude. Finally, the temperature change is the third source of fault. The temperature is highly related to the viscous friction, which is the main friction factor of the wheel. The temperature fault will cause the wheel to operate in an abnormal condition. Model-based methods are a number of methods based on intelligent and learning-based strategies [3], [4], [5]. These methods make use of the large amount of process history data. Fuzzy logic technique has been investigated as powerful decision making tool as they can be used as supervisory schemes to make the fault analysis decisions [6], [7]. The basic idea behind the model based observer approaches is to estimate the states of the system by using either Linear or Nonlinear observers. The state estimation error is served as the residual. The advantage of using the observer is the flexibility to select its gains that leads to a rich variety of FDI schemes [8], [9].

In this paper, a nonlinear observer is employed for the reaction wheel of each axis so as to observe the estimated angular velocity and the motor current from each wheel. Among these estimated signals, one will be able to identify the existence and isolation of faults in the system. The outline of the remainder of this paper is as follows. In Section 2, contribution to sustainability is defined. Section 3, a brief review of the attitude control system and model of the reaction wheel will be given. Section 4 presents results for a nonlinear observer-based scheme used for fault detection. Section 5 presents results for a fuzzy-based scheme used for fault isolation. In Section 6, by combining two previous sections a nonlinear-fuzzy based FDI scheme will be developed. A comparative study between nonlinear-fuzzy and linear observer based FDI scheme will be conducted in Section 7. These results will serves as benchmark data for comparison with the proposed FDI scheme. These comparative results will demonstrate the advantages of nonlinear-fuzzy based scheme developed in this paper.

\section{Contribution to Sustainability}

The whole observation and decision making process is done by accurate computer systems and this insures the ability of the system to survive without human interference. The proposed method used for attitude control system is innovative and the results of that are very satisfactory and suitable to implementation and test on the real reaction wheel.

\section{Reaction Wheel Model}

A reaction wheel consists of a rotating flywheel, typically suspended on ball bearings, and driven by an inertial brushless DC motor. Fig.1 provides the fundamental relationships for a high fidelity mathematical model of a reaction wheel system. There are five main sub-blocks in the diagram: motor torque control, speed limiter, EMF torque limiting, motor disturbances and bearing friction and disturbances. The reaction wheel applied in this paper is the ITHACO's standard Type A. Its typical parameter values used can be found in [3]. Operating in space a satellite experiences many types of external environmental disturbance torques. Four main disturbances we considered here are: gravitation torque, solar pressure torque, magnetic torque and aerodynamic torque. It is assumed that the maximum external disturbance torque is the sum of these four maximum torques: 


$$
\mathrm{DIS}=\mathrm{DIS}_{\mathrm{gg}}+\mathrm{DIS}_{\mathrm{sp}}+\mathrm{DIS}_{\mathrm{mf}}+\mathrm{DIS}_{\mathrm{ad}}=5.68 \times 10^{-5} \mathrm{~N}-\mathrm{m}
$$

We assume that the external disturbance torque is a normally distributed random signal with zero mean and variance:

$$
\operatorname{DIS}^{2}=\left(5.68 \times 10^{-5}\right)^{2}[2]
$$

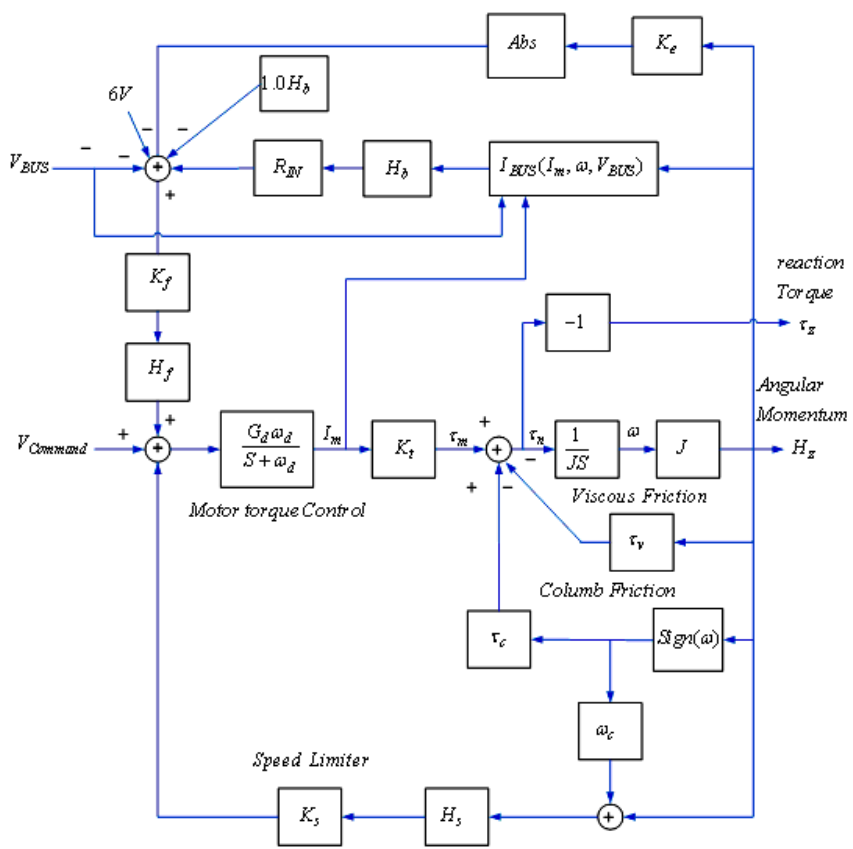

Fig. 1. High Fidelity Reaction Wheel Block Diagram

\section{Nonlinear Observer-Based Fault Detection}

FDI for nonlinear systems can be achieved by generating residuals using nonlinear observers. The nonlinear observer based residual generation problem can be formulated as follows; consider a nonlinear system given by (1).

$$
\left\{\begin{array}{l}
\dot{\mathrm{x}}=\mathrm{f}(\mathrm{x}, \mathrm{u}) \\
\mathrm{y}=\mathrm{h}(\mathrm{x}, \mathrm{u})
\end{array}\right.
$$

Under the assumption of a smooth system [10], and assuming that $\mathrm{H}$ defined below is full rank, a simple form of a nonlinear estimator described by (2).

$$
\left\{\left.\begin{array}{l}
\dot{x}=f(x, u)+L(\hat{x}, u)(y-\hat{y}), \quad H(\hat{x}, u)=\frac{\partial \hat{f}}{\partial y} \\
y=h(\hat{x}, u)
\end{array}\right|_{\hat{x}, u}\right.
$$

Where $\mathrm{H}$ is a time varying observer gain matrix; the state estimation error equation and the output estimation error then are: 


$$
\left\{\begin{array}{l}
\dot{e}(t)=\left[\frac{\partial \hat{f}}{\partial y}-H(x, u) \frac{\partial h}{\partial x}\right]_{\hat{x}, u} e(t) \\
e(t)=y(t)-\hat{y}(t)=h(x, u)-h(\hat{x}, u)
\end{array}\right.
$$

The nonlinear system residual generator using a nonlinear observer is illustrated in Fig. 2.

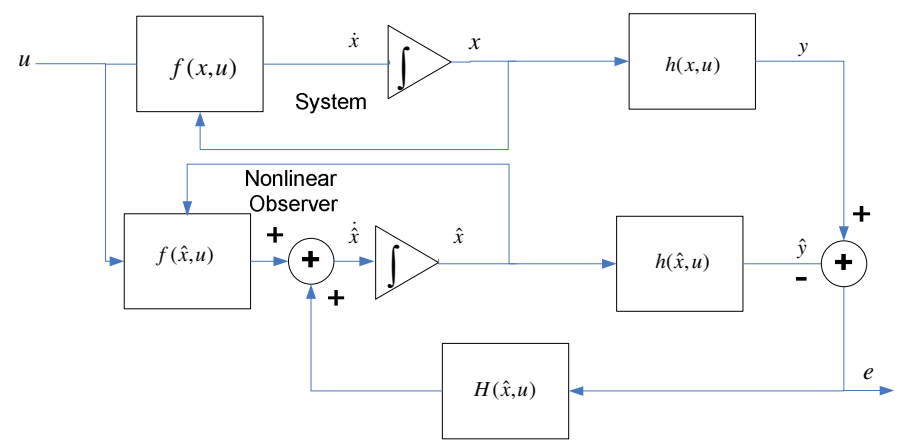

Fig. 2. Nonlinear Observer Residual Generator

The two variables $\omega$ and $I_{m}$ in the reaction wheel model have been selected as state variables.

It is assumed that both these state variables are reachable.

Dynamic equations of the reaction wheel based on equation (4) are determined [11]. In this equation, $f_{1}, f_{2}$ are the functions for modeling motor disturbances, $f_{3}$ is output of EMF Torque Limiting block, $f_{4}$ is a sigmoidal function for modeling coulomb friction and $f_{5}$ represents the speed limiter block. $n$ is the torque noise and $r$ is the reference input or the torque command [11], [12].

$$
\begin{aligned}
& \dot{\omega}=\frac{1}{J}\left[f_{1}(\omega)+k_{t} I_{m}\left[f_{2}(\omega)+1\right]-\tau_{v} \omega-\tau_{c} f_{4}(\omega)+n\right] \\
& \dot{I}_{m}=G_{d} \omega_{d}\left[f_{3}\left(\omega, I_{m}\right)-f_{5}(\omega)\right]-\omega_{d} I_{m}+G_{d} \omega_{d} r
\end{aligned}
$$

\section{Fuzzy-Based Fault Isolation}

After generation of residual Signals, the second step is decision making based on these residuals. At this step, given the characteristics of the two residuals, there should be three types of fault correctly isolated. Fuzzy logic as a powerful tool in inference and decision making based on linguistic terms and if-then rules used to isolate the three types of faults in the reaction wheel; Takagi-Sugeno, newer method of fuzzy inference than Mamdani Introduced in 1985 [13], is similar to the Mamdani method in many aspects. The first two parts of the fuzzy inference process, fuzzifying the inputs and applying the fuzzy operator, are exactly the same. The main difference between Mamdani and Sugeno is that the Sugeno output membership functions are either linear or constant. Based on this, Takagi-Sugeno is more suitable than other fuzzy implication methods. General Residual fuzzy evaluation scheme is illustrated in Fig. 3. 


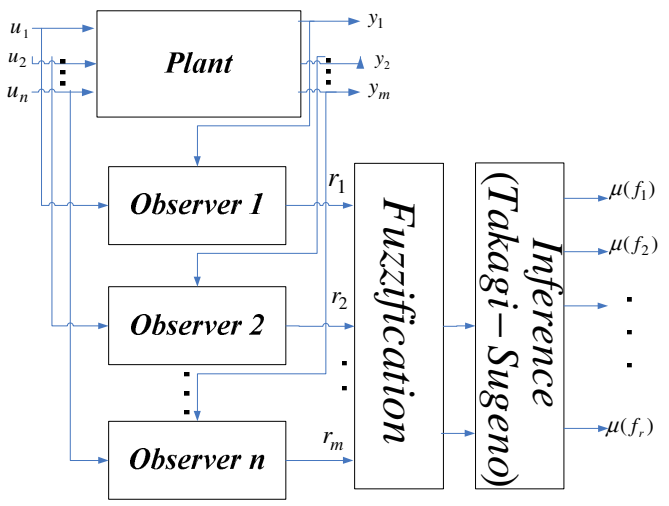

Fig. 3. Residual fuzzy evaluation

Where $u_{i}, \mathrm{i}=1,2, \ldots, n$ are the inputs, $y_{i}, \mathrm{i}=1,2, \ldots, m$ are the outputs, $r_{i}, \mathrm{i}=1,2, \ldots, m$ are the residuals, and $\mu\left(f_{r}\right)$ is the possibility of each hypothesized fault. Fig. 4 shows the fuzzification of the two residuals obtained in the previous section, the angular velocity and the motor current. Although the two residuals is not independent manner to the three types of faults, but using five membership functions this goal is attained. Each input in NB, NS, Z, PS and PB respectively indicating Negative Big, Negative Small, Zero, Positive Small and Positive Big. Intermediate membership functions are of Gaussian type and membership functions at the beginning and end, are respectively of $\mathrm{S}$ and $\mathrm{Z}$ type.
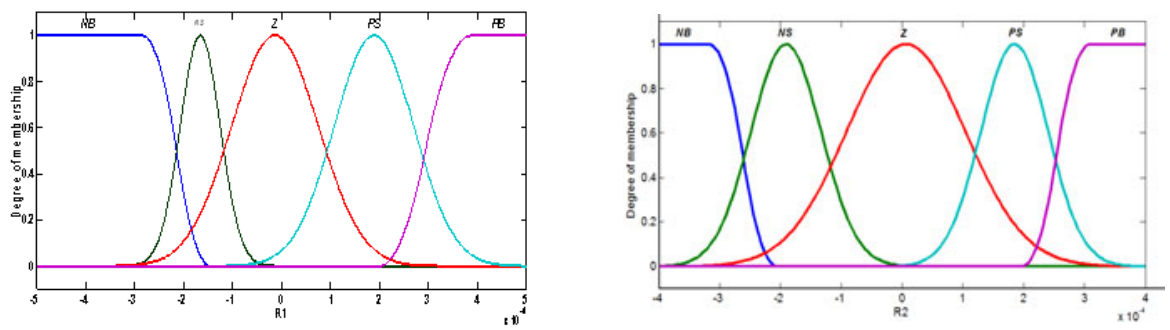

Fig. 4. Angular velocity $\left(r_{1}\right)$, Motor current $\left(r_{2}\right)$ membership Functions

For inference we can define:

$$
\left\{\begin{array}{l}
\mu_{r_{1} \cap r_{2}}\left(f_{r}\right)=\mu_{r_{1}}\left(f_{r}\right) * \mu_{r_{2}}\left(f_{r}\right) \\
\mu_{r_{1} \cup r_{2}}\left(f_{r}\right)=\mu_{r_{1}}\left(f_{r}\right)+\mu_{r_{2}}\left(f_{r}\right)-\mu_{r_{1}}\left(f_{r}\right) \mu_{r_{2}}\left(f_{r}\right): x \in X \rightarrow[0,1]
\end{array}\right.
$$

In general, the number of rules derived from two inputs, to each output is 25 . But according to the goal, and considering the simplicity and optimality of the design, based on the research done on the system and using the table 1 only 10 fuzzy rules are enough. Temp, Vbus and Im-Fault are outputs of the decision making unit. The output value is zero or one. Zero means a normal condition and one refers to a fault condition. 
Table 1. Relationships between two residuals and three Faults

\begin{tabular}{|c|c|c|c|c|c|}
\hline & NB & NS & $\mathbf{Z}$ & PS & PB \\
\hline NB & 1 & 0 & O & 0 & 0 \\
\hline NS & 1 & 0 & 0 & 0 & 0 \\
\hline \begin{tabular}{|l|}
$\mathbf{Z}$ \\
\end{tabular} & 1 & 0 & 0 & 0 & 0 \\
\hline PS & 1 & 0 & 0 & 0 & 0 \\
\hline PB & 1 & 0 & 0 & 0 & 0 \\
\hline
\end{tabular}

Voltage Bus Fault

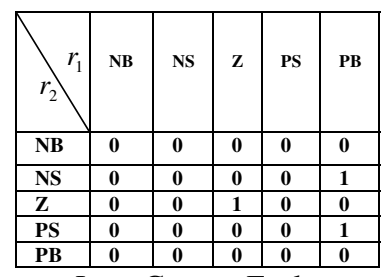

Loss Current Fault

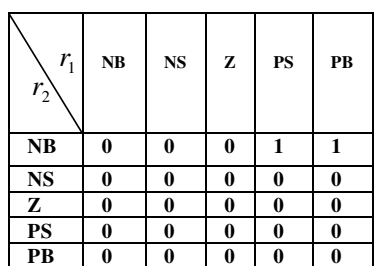

Temperature Fault

Combining these three tables, following rules can be obtained.

Rule 1 : if $r_{1}=N B$ AND $r_{2}=N B$ Then Vbus $F=1 A N D \operatorname{Im} F=0 A N D T e m p F=0$

Rule 2: if $r_{1}=N S$ AND $r_{2}=N B$ Then VbusF $=1 A N D \operatorname{Im} F=0 A N D T e m p F=0$

Rule 10 : if $r_{1}=N B$ AND $r_{2}=P B$ Then Vbus $F=0 A N D \operatorname{Im} F=0 A N D T e m p F=1$

These rules are set in such a way that all three types of fault can be isolated into a wide range of operating points of attitude control system. In Takagi-Sugeno fuzzy decision making scheme, the weighted-average method has been used for defuzzification.

\section{Simulation Results}

When the normal value of the bus voltage (which is $8 \mathrm{~V}$ ) drops, the motor torque may be limited at high speeds due to the increasing back-EMF of the motor, and this eventually results in a reduced torque capacity of the wheel. When this value becomes too low (e.g. 3V), the attitude control system will malfunction and the attitude the spacecraft becomes unstable. Similarly, since the motor torque is directly related to the motor current through one constant parameter $k_{t}$, when some kind of motor current loss occurs in the reaction wheel, the motor torque will drop down accordingly. Therefore, the wheel can no longer supply enough motor torque to the attitude control system. When the current loss becomes significant, the controlled attitude angle will become unstable. It is well-known that viscous friction is present in the bearings due to the bearing lubricant. When the bearings are damaged seriously, this viscous friction becomes much larger than that when it is in normal conditions. Since the temperature of the wheel is strongly related to the viscous friction in the wheel, this suggests that an estimate of the working condition of the bearings is possible through monitoring wheel temperature.

\subsection{Bus Voltage Fault}

Fig. 5 shows a case study of the three faults. As seen, the bus voltage of the reaction wheel aligned on the $\mathrm{X}$ axis has dropped from the normal value of $8 \mathrm{~V}$ to $5.5 \mathrm{~V}$ in 200 seconds. After few seconds, bus voltage fault was detected. Hence, the bus voltage fault in the wheel of the $\mathrm{X}$ axis is detected and isolated correctly. 


\subsection{Current Loss Fault}

Similarly, a current loss fault in the wheel of the $\mathrm{X}$ axis is properly detected and isolated as shown in Fig. 5. The current limiter signal of the wheel on the $\mathrm{X}$ axis has dropped from 1 to 0.40 in 100 seconds, implying that $60 \%$ of the motor current has been lost. Accordingly, the residual signal of the $\mathrm{X}$ axis has increased that output of fuzzy decision making unit is one after a short time delay and this indicated that the wheel on the $\mathrm{X}$ axis is faulty.

\subsection{Temperature Fault}

The simulations in Fig. 5 have illustrated that the proposed nonlinear-fuzzy scheme is also effective in detecting and isolating a temperature fault that has occurred in the wheel on the $\mathrm{X}$ axis at 100 seconds. Note that in each of the three cases, the residual curves for the other wheels will remain below their threshold.

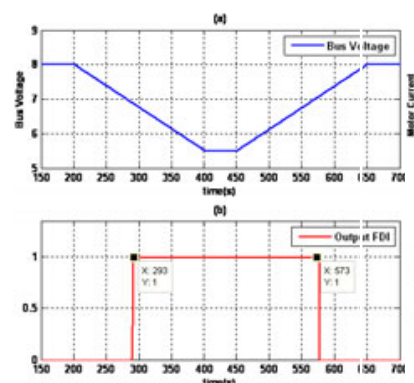

Voltage Bus Fault

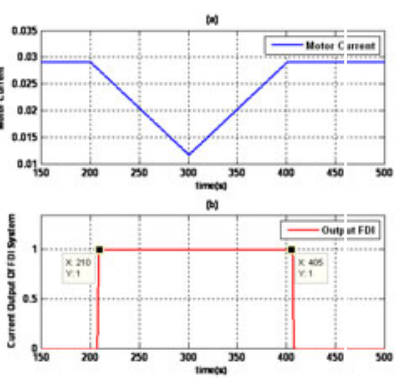

Motor Current Fault Isolation

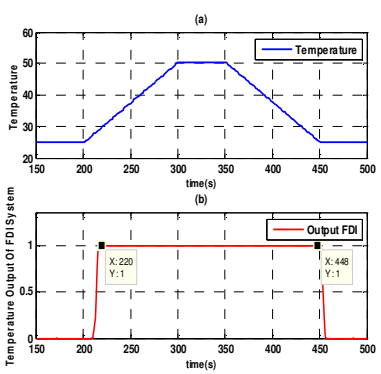

Temperature Fault Isolation

Fig. 5. Three Fault Isolation and their sensitivity to changes of voltage, current, temperature

\section{Comparison of Linear and Nonlinear-Fuzzy Based FDI Schemes}

The success of the two FDI schemes success highly depends on the modeling accuracy of the reaction wheel. As seen in Fig.1, reaction wheel is a highly nonlinear dynamic system. It contains many nonlinear elements and disturbances. For linear observer-based FDI scheme, one has to omit all these nonlinearities and disturbances effects and only use a linear model to observe the dynamics of the linearized reaction wheel model. In this paper, the objective is to design a fault diagnosis algorithm for the nonlinear model as the linear observer estimates are less likely to converge to states of a nonlinear modẹl(not shown due to space limitations). With this in mind, a nonlinear observer is designed to improve the possible inefficiencies of a linear fault diagnosis observer. Choosing the nominal voltage bus that places the system in a less nonlinear area, this ensures that a linear observer is able to estimate to original nonlinear system.it could be shown that nevertheless it does not usually provide accurate information about the state of the system during the presence of faults. Thus it is not a good candidate for fault diagnosis in high nonlinear area. 


\section{Conclusion}

This paper has developed and presented a nonlinear-fuzzy based scheme for fault detection and isolation in reaction wheels of a satellite. Faults considered are the bus voltage fault, the motor current loss fault and the temperature fault. First, we investigated a linear diagnosis scheme for fault detection and isolation. It is shown that this method has a capability for fault detection and isolation in some cases, but it is not reliable and useful under all operating conditions of the system. Since linear observer was not successful in detecting all types of faults, the performance of a nonlinear observer designed was shown that this observer can outperform the linear observer and detect all types of faults under different operating conditions of the system. Subsequently, a nonlinear-fuzzy based scheme is introduced to achieve better FDI performance of the reaction wheels. The proposed nonlinear-fuzzy based scheme consists of three networks applied to estimate the states of the wheels in three axes separately and to simplify the fault detection and isolation process. Through a comparative study between a linear and nonlinear-fuzzy scheme, it is demonstrated that the latter's performance is superior to the former's for FDI.

\section{References}

1. Wertz, J.R. (ed.): Spacecraft Attitude Determination and Control. Kluwer Academics Publishers, Dordrecht (1995)

2. Bialke, B.: High Fidelity Mathematical Modeling of Reaction Wheel Performance. Advances in Astronautical Sciences 98, 483-496 (1998)

3. Venkatasubramanian, V., Rengaswamy, R., Yin, K.: A review of process fault detection and diagnosis Part I: Quantitative model-based methods. Computers and Chemical Engineering (2002)

4. Isermann, R.: Fault-Diagnosis Systems: An Introduction from Fault. Detection to Fault Tolerance. Springer, Berlin (2006)

5. Simani, S., Fantuzzi, C., Patton, R.J.: Model-based Fault Diagnosis in Dynamic Systems Using Identification Techniques, February 18. Springer, Heidelberg (2004)

6. Palade, V., Bocaniala, C.D., Jain, L. (eds.): Computational Intelligence in Fault Diagnosis. Springer, London (2006)

7. Soliman, A.A.: The Application of Fuzzy Logic to the Diagnosis of Automotive Systems. The Ohio State University (1997)

8. Frank, P.M.: Fault Diagnosis in dynamic systems using analytical and knowledge-based redundancy-A survey. Automatica 26, 459-474 (1990)

9. Frank, P.M.: Enhancement of Robustness on Observer-Based Fault Detection. International Journal of Control, 59(4), 955-983

10. Isidori, A.: Nonlinear Control System: An Introduction. Springer, New York (1989)

11. Azarnoush, H., Khorasani, K.: Fault Detection in Spacecraft Attitude Control System. IEEE, Los Alamitos (2007)

12. Li, Z.Q., Ma, L., Khorasani, K.: Fault Diagnosis of an Actuator in the Attitude Control Subsystem of a Satellite using Neural Networks. In: Proceedings of International Joint Conference on Neural Networks, Orlando, Florida, USA (2007)

13. Sugeno, M.: Industrial applications of fuzzy control. Elsevier Science Pub. Co., Amsterdam (1985) 